Proceedings of the 16th Czech and Slovak Conference on Magnetism, Košice, Slovakia, June 13-17, 2016

\title{
Angular Dependence of Magnetization Reversal in Microwire Studied by MOKE
}

\begin{abstract}
O. VÁHOVSKÝ* AND R. VARGA
Institute of Physics, Faculty of Sciences, P.J. Safarik University, Park Angelinum 9, 04154 Košice, Slovakia

In this work we present magneto-optical Kerr effect study of magnetization processes in amorphous glass coated microwire of composition $\mathrm{Fe}_{77.5} \mathrm{Si}_{7.5} \mathrm{~B}_{15}$. Unique results obtained from microwire were compared to results of similar measurements on polycrystalline ribbon $\mathrm{Co}_{2} \mathrm{FeSi}$. In both cases reversal of longitudinal and transversal component of surface magnetization was studied. There was just a small variation in total change of magnetization caused by change in the direction of magnetic field in case of square hysteresis loops measured on microwire. However, switching field was angularly dependent and the change was considerably high and well predictable. The last two facts make such microwires outstanding candidates for sensors of magnetic field direction or spatial orientation.
\end{abstract}

DOI: 10.12693/APhysPolA.131.648

PACS/topics: $75.50 . \mathrm{Kj}, 75.60 . \mathrm{Ej}$

\section{Introduction}

Controlled manipulation of a single domain wall has become of great interest in the light of possible applications, e.g. domain-wall based logic devices or magnetic memories $[1,2]$. Classically, magnetic properties of microwires are measured by induction methods $[3,4]$. However, numerous problems appear due to the relatively large relaxation time of pick-up coils when very fast domain wall needs to be studied. In such cases, application of the Kerr-effect based measuring methods is desirable. Magneto-optical methods present a way of nondestructive, contactless measurement of magnetic properties and are successfully used to study various properties of micro- and nanomaterials [5-7]. It is mostly due to their ability of detecting very small changes of magnetization, even if they happen over a very short period of time. As against induction methods, magneto-optical Kerr effect (MOKE) methods have much shorter relaxation times and the results are comparable.

In the given contribution, we present the Kerr-effect study of magnetization processes in magnetic microwires under magnetic field applied in different angles $\phi$ with regard to wire axis. Variation in total magnetization change is negligible, while strong dependence of switching field on $\phi$ is observed.

Similar measurements were performed on polycrystalline ribbon to emphasize unique magnetic properties of the microwire surface. Both surface of the microwire and surface of the ribbon have multidomain structure. In case of the ribbon, magnetic state on the surface depends mainly on the external magnetic field. On the other hand, magnetization on the surface of microwire is influenced not only by the external magnetic field, but also by the magnetization of inner axial domain.

\footnotetext{
* corresponding author; e-mail: oliver.vahovsky@student.upjs.sk
}

\section{Experimental}

Glass-coated $\mathrm{Fe}_{77.5} \mathrm{Si}_{7.5} \mathrm{~B}_{15}$ microwire prepared by the Taylor-Ulitovski method was used for measurements. The sample was $3 \mathrm{~cm}$ long with diameter of metallic core $14 \mu \mathrm{m}$ and glass coat width of $7.5 \mu \mathrm{m}$. Metallic core has amorphous structure (Fig. 1a). It is well known that domain structure of an amorphous glasscoated microwire consists of bistable axially magnetized inner core and a surface shell with generally different direction of magnetization compared to the core. Magnetostatic energy is minimized by closure domains on both ends of the microwire (Fig. 1c). a)

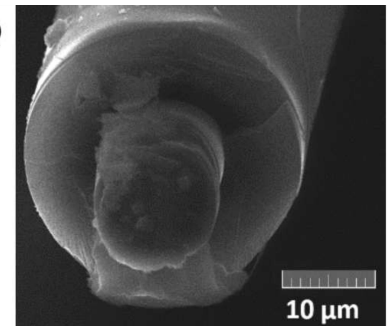

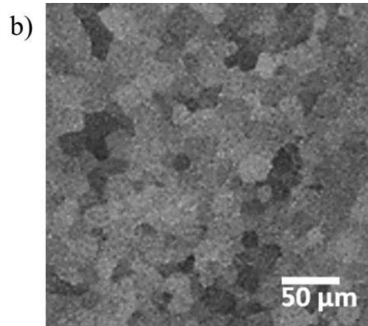

c)

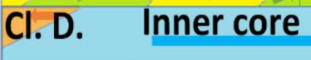

Outer Shell

Fig. 1. a) SEM image of $\mathrm{Fe}_{77.5} \mathrm{Si}_{7.5} \mathrm{~B}_{15}$ microwire, b) SEM image of $\mathrm{Co}_{2} \mathrm{FeSi}$ ribbon surface, c) Schematic picture of microwire domain structure (Cl. D. = closure domain).

MOKE results from microwire were compared to similar measurements on polycrystalline ribbon. $\mathrm{Co}_{2} \mathrm{FeSi}$ ribbon was prepared by melt spinning. Size of the ribbon specimen was $8 \mathrm{~mm} \times 3 \mathrm{~mm}$ with width of $60.6 \mu \mathrm{m}$. Polycrystalline character of the sample is clearly visible on SEM pictures (Fig. 1b) and implies multidomain structure on the surface of the ribbon. 
MOKE hysteresis loops were measured in 2D magnetic field with amplitude of $3000 \mathrm{~A} / \mathrm{m}$. Voltage input signal was sine with frequency $40 \mathrm{~Hz}$. The field was produced by 4 solenoids placed in a cross shape, effectively capable of producing magnetic field in any direction in the plane of solenoids. It was in a vertical position, such that incident plane for laser beam $(\lambda=633 \mathrm{~nm})$ is horizontal. Solenoids with shared axis were both fed by a single current source. Amplitude and start phase were properly set (two-channel generator was used). The reflection from the illuminated part of the wire was captured by Si photodetector. The change of longitudinal as well as transversal component of surface magnetization was studied. Although both specimens have multidomain surface structure, they behave differently under the effect of external magnetic field. Polarizer was used when measuring the reversal of longitudinal component of magnetization. MOKE signal as well as voltage in solenoids over time was displayed in real time on oscilloscope. Simplified scheme of the experimental setup is in Fig. 2.

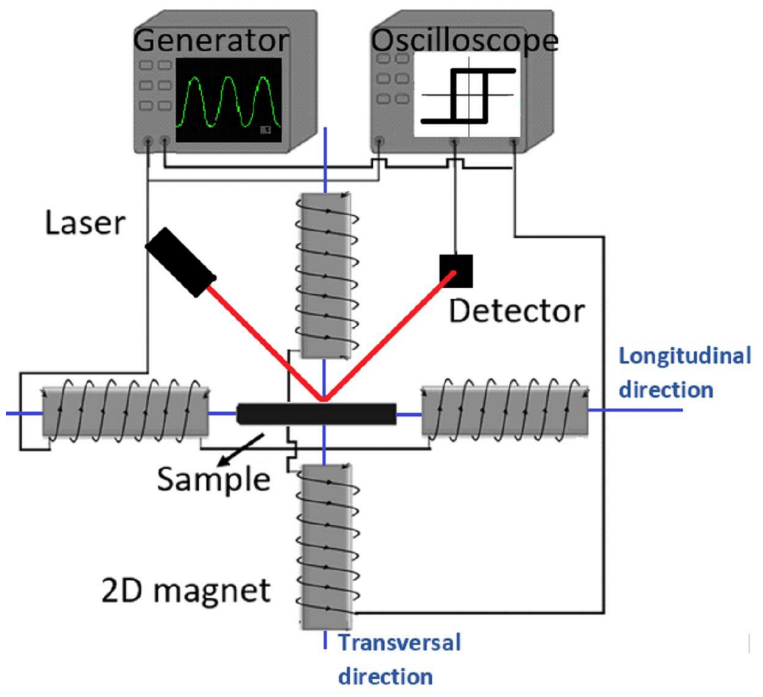

Fig. 2. Simplified scheme of our MOKE setup.

\section{Results and discussion}

Let $\phi$ be the angle between magnetic field direction and the axis of the specimen. For both ribbon and microwire, hysteresis loops for field applied in $\phi$ ranging from $0^{\circ}$ to $360^{\circ}$ (by $10^{\circ}$ steps) were measured. Hysteresis loops of ribbon present continuous reversal of magnetization, indicating magnetization processes most probably consisting of domain wall movement as well as rotation of magnetization (Fig. 3). Total change in magnetization during reversal $\Delta M$ depends on $\phi$ (minor hysteresis loops are observed). Longitudinal magnetization change is proportional to $|\cos (\phi)|$, while transversal magnetization change to $|\sin (\phi)|$ (Fig. 4). This indicates easy plane anisotropy on the surface of the ribbon.

On the contrary, square hysteresis loops are observed in the case of microwire. Very small variation of total

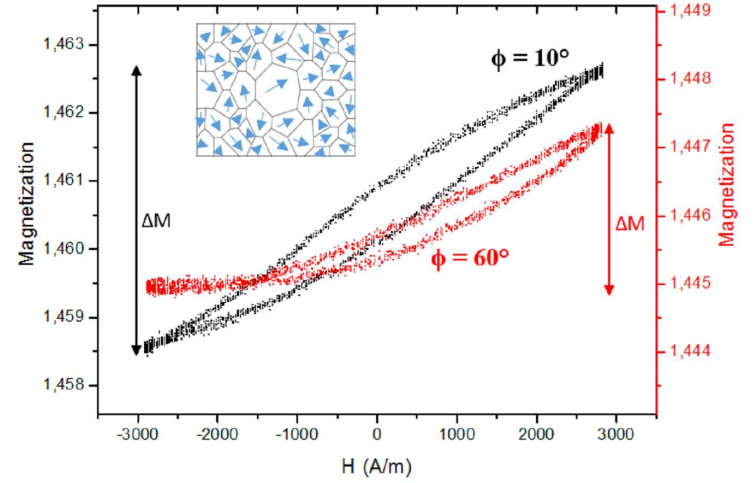

Fig. 3. Hysteresis loops for change of longitudinal magnetization of a $\mathrm{Co}_{2} \mathrm{FeSi}$ ribbon.

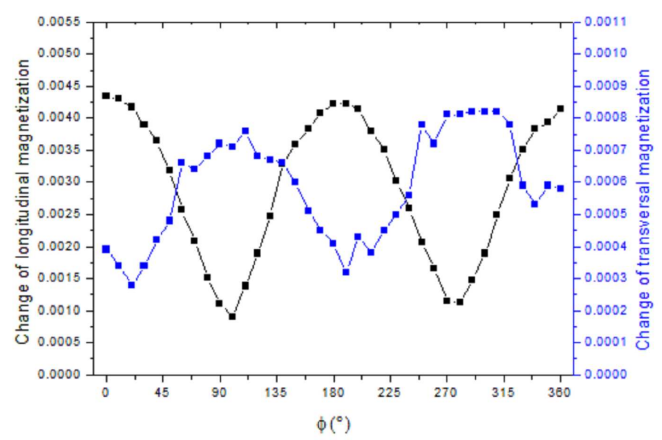

Fig. 4. Angular dependence of total change of magnetization for $\mathrm{Co}_{2} \mathrm{FeSi}$ ribbon.

magnetization change is observed. These results are consistent with its bistable character and magnetization reversal in one large Barkhausen jump [8] (Fig. 5). Precisely at $90^{\circ}$ and $270^{\circ}$ no domain wall jump occurs (field is applied along the hard axis of the microwire). However, dependence of switching field on $\phi$ is apparent. Only longitudinal component of switching field $\left(H_{s w}\right)$ is responsible for the Barkhausen jump.

For jump to happen that component has to be equal to switching field at $\phi=0^{\circ}$, i.e.

$$
H_{s w}|\cos \phi|=\left|H_{s w}\left(0^{\circ}\right)\right| \text {, }
$$

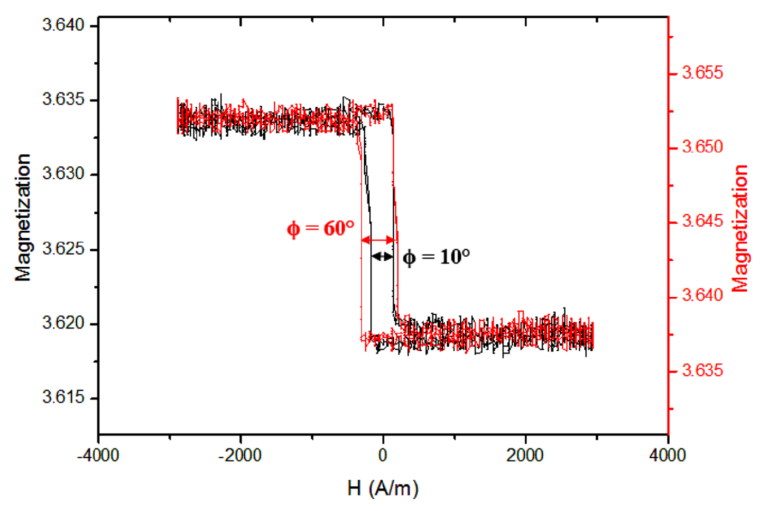

Fig. 5. Hysteresis loops of a $\mathrm{Fe}_{77.5} \mathrm{Si}_{7.5} \mathrm{~B}_{15}$ microwire. 


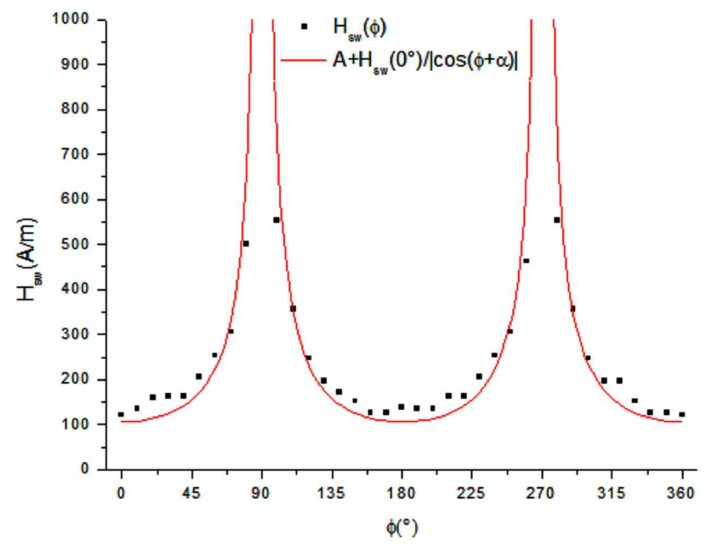

Fig. 6. Angular dependence of switching field for $\mathrm{Fe}_{77.5} \mathrm{Si}_{7.5} \mathrm{~B}_{15}$ microwire.

$$
H_{s w}=\frac{\left|H_{s w}\left(0^{\circ}\right)\right|}{|\cos \phi|} .
$$

The dependence of switching field on $\phi$ is in Fig. 6. Qualitatively it agrees very well with the equation above.

If the specimen's easy direction of magnetization is unknown, angular dependence of switching field can be fitted as

$$
H_{s w}=A+\frac{\left|H_{s w}\left(0^{\circ}\right)\right|}{|\cos (\phi+\alpha)|}
$$

where $\alpha$ determines the angle between easy direction of magnetization and horizontal direction. In our case $\alpha<1^{\circ}$, which is in good agreement with axial direction of magnetization in the inner core of microwire. We cannot find the angle of magnetization in outer shell with this method for such microwires, because reversal of surface magnetization is caused by interaction with domain wall propagating through the inner core [8].

In the case when the direction of external AC field is unknown, it can be found from the measured value of $H_{s w}$ (if the angular dependence was calibrated before). Hence it is easy to determine the unknown direction of external AC field. Or, other way round, the unknown direction of microwire with regard to known direction of field. Consequently, bistable microwires can be used as sensors of AC magnetic field direction or sensors of spatial orientation.

\section{Conclusions}

Here we study angular dependence of magnetization reversal in magnetic microwire $\mathrm{Fe}_{77.5} \mathrm{Si}_{7.5} \mathrm{~B}_{15}$ and compare some of the results to similar study of polycrystalline ribbon $\mathrm{Co}_{2} \mathrm{FeSi}$. Surface domain structure of the ribbon is mainly affected by the external magnetic field. Magnetization reversal consists of movement of a number of domain walls and considerably high magnetic field is needed to acquire saturated state.
On the other hand, abrupt change of magnetization is observed on the surface of microwire. This change does not depend on the direction of magnetic field. The reason is that surface shell is subjected to superposition of the external magnetic field and magnetization of the inner core. As a result, surface shell is magnetically bistable and easily saturated along its easy axis of magnetization although there is much stronger anisotropy than on the ribbon surface (switching field $=$ saturation field is $<130 \mathrm{~A} / \mathrm{m}$ ). Switching field strongly depends on the direction of magnetic field with respect to the wire axis.

Such dependence can be used for construction of AC magnetic field direction sensor or spatial orientation sensor.

\section{Acknowledgments}

This work was supported by the projects APVV-002711 and Slovak VEGA grant No. 1/0164/16.

\section{References}

[1] J.A. Currivan-Incorvia, S. Siddiqui, S. Dutta, E.R. Evarts, J. Zhang, D. Bono, C.A. Ross, M.A. Baldo, Nat. Commun. 7, 10275 (2016).

[2] S.S.P. Parkin, M. Hayashi, L. Thomas, Science 320 , 190 (2008).

[3] J. Ziman, J. Onufer, M. Kladivová, J. Magn. Magn. Mater. 323, (2011).

[4] J. Onufer, J. Ziman, M. Kladivová, J. Magn. Magn. Mater. 396, (2015).

[5] C. Favieres, C. Aroca, M.C. Sánchez, V. Madurga, J. Appl. Phys. 91, 9995 (2002).

[6] A. Chizhik, C. Garcia, J. Gonzales, J.M. Blanco, J. Magn. Magn. Mater. 279, 359 (2004).

[7] A. Chizhik, A. Zhukov, J.M. Blanco, J. Gonzales, J. Magn. Magn. Mater. 324, 3563 (2012).

[8] K. Richter, A. Thiaville, R. Varga, IEEE Trans. Magn. 50, (2014). 